Review

\title{
Supersymmetric Extensions of Non-Relativistic Scaling Algebras
}

Makoto Sakaguchi ${ }^{1}$ and Kentaroh Yoshida ${ }^{2, *}$

${ }^{1}$ Department of Physics, Ibaraki University, Mito 310-8512, Japan;

E-Mail: msakaguc@mx.ibaraki.ac.jp

${ }^{2}$ Department of Physics, Kyoto University, Kyoto 606-8502, Japan

* Author to whom correspondence should be addressed;

E-Mail: kyoshida@gauge.scphys.kyoto-u.ac.jp; Tel.: +81-75-753-3879; Fax: +81-75-753-3886.

Received: 3 July 2012; in revised form: 7 August 2012 / Accepted: 8 August 2012 /

Published: 24 August 2012

\begin{abstract}
An exciting subject in string theory is to consider some applications of the AdS/CFT correspondence to realistic systems like condensed matter systems. Since most of such systems are non-relativistic, an anisotropic scaling symmetry with the general value of dynamical critical exponent $z$ plays an important role in constructing the gravity duals for non-relativistic field theories. Supersymmetric extensions of symmetry algebras including the anisotropic scaling are very helpful to consider holographic relations accurately. We give a short summary on the classification of superalgebras with the anisotropic scaling as subalgebras of the following Lie superalgebras, $\operatorname{psu}(2,2 \mid 4)$, osp $(8 \mid 4)$ and osp $\left(8^{*} \mid 4\right)$, which appear in the study of AdS/CFT in type IIB string and M theories. It contains supersymmetric extensions of Schrödinger algebra and Lifshitz algebra.
\end{abstract}

Keywords: supersymmetry; non-relativistic limit; scale invariance; AdS/CFT

\section{Introduction}

One of the most well-studied subjects in string theory is the AdS/CFT correspondence [1-3]. Although it has not been rigorously proven yet, it is supported by an enormous amount of evidence and there is no doubt for the correspondence. Assuming that it surely holds, there are two important assets. The one is that AdS/CFT provides us a powerful tool to study the unknown quantum gravity from the well-known quantum field theory without gravity. The other is that the AdS/CFT correspondence is a 
strong/weak duality and it enables us to study strongly-coupled quantum field theories non-perturbatively by using a description of classical gravitational theories.

Based on the latter aspect, many applications of AdS/CFT to realistic systems in nature like QCD and condensed matter physics have been explored enthusiastically (for comprehensive reviews, for example, see $[4,5])$. In this direction there is a motive to figure out the holographic description of non-relativistic field theories because most condensed matter systems are non-relativistic. In particular, having some applications of AdS/CFT to condensed matter systems in our mind, we are interested in non-relativistic fixed points. For example, such fixed points appear in real experiments using ultra-cold atoms. The fixed points exhibit an anisotropic scaling invariance defined by a dynamical critical exponent $z$ like

$$
t \rightarrow \lambda^{z} t, \quad x^{i} \rightarrow \lambda x^{i} \quad(i=1, \ldots, d)
$$

where $\lambda$ is a scaling constant and $d$ is the number of spatial directions. The exponent $z$ measures the anisotropy in the time direction $t$. When $z=1$, this is the usual scaling symmetry in relativistic field theories. The case with $z \neq 1$ does not respect the Lorentz symmetry any more and the system has to be realized in a non-relativistic manner. The invariance under the anisotropic scaling (1) is a key ingredient to construct the spacetime metrics of the gravity duals [6-8]. Then the spacetimes described by these metrics are homogeneous and are represented by cosets [9]. Thus it is of importance to consider symmetry algebras with an isotropic scaling invariance like conformal symmetries in conformal field theories. (For example, the Schrödinger symmetry [10,11] fixes the behavior of two-point functions [12,13].) The scaling symmetry provides us a first clue in looking for the holographic description as in the usual study of AdS/CFT.

There are two typical examples of algebras including a non-relativistic scale invariance with $z \neq 1$. The former is the Schrödinger algebra [10,11] and the latter is the Lifshitz algebra (For the explicit algebra, e.g., see $[6,7,9,13])$. The Schrödinger algebra comprises the centrally extended Galilean (Bargmann) algebra and the dilatation with $z \neq 1$. (Rigorously speaking, the $z=2$ case is called the Schrödinger algebra and then the generator of special conformal transformation is contained. However, for convenience, we will call the algebra with $z \neq 1$ the Schrödinger algebra with $z$ loosely as in most of the recent works.) The Lifshitz algebra consists of time and spatial translations, spatial rotations and the dilatation with $z \neq 1$ (in particular, no Galilean boost). It is well known that the two algebras can be realized as subalgebras of relativistic conformal algebras $(z=1)$ and it would be helpful to see a schematic sequence of the algebras like

\section{Lifshitz algebra $\subset$ Schrödinger algebra $\subset$ Relativistic conformal algebra.}

Thus the non-relativistic algebras are intimately related each other from the point of view of the algebraic structure.

The purpose of this review article is to give a short summary on the classification of superalgebras with the anisotropic scaling (1) as subalgebras of the following Lie superalgebras (for other Lie superalgebras, see earlier works [14,15]), psu(2,2|4), osp(8|4) and osp $\left(8^{*} \mid 4\right)$, which are concerned with AdS/CFT in type IIB string and $\mathrm{M}$ theories. It contains supersymmetric extensions of Schrödinger algebra and Lifshitz algebra. This classification is basically based on the previous works [16,17] but it contains a generalization of the results $[16,17]$ to the arbitrary $z$ case and a new result on supersymmetric Lifshitz algebras. 
This review article is organized as follows. In Section 2 we give general prescriptions to pick up a subalgebra including an anisotropic scaling. In Section 3 possible superalgebras including the anisotropic scaling invariance are classified as subalgebras of psu(2,2|4). In Sections 4 and 5 we classify superalgebras of $\operatorname{osp}(8 \mid 4)$ and $\operatorname{osp}\left(8^{*} \mid 4\right)$ in the same way. Section 6 is devoted to summary. In Appendices we summarize the notation and convention of $\operatorname{psu}(2,2 \mid 4), \operatorname{osp}(8 \mid 4)$ and $\operatorname{osp}\left(8^{*} \mid 4\right)$ utilized in this article.

\section{General Prescriptions}

We explain general prescriptions to pick up a subalgebra with an anisotropic scaling in order to make our discussion clear.

The first is a prescription to pick up subalgebras. As a warm-up, let us consider a relativistic conformal algebra in four dimensions, that is a portion of the bosonic part of $\operatorname{psu}(2,2 \mid 4)$,

$$
\left\{P_{\mu}, L_{\mu \nu}, D, K_{\mu}\right\} \quad(\mu, \nu=0,1,2,3)
$$

Here $P_{\mu}$ describes a time translation and spatial translations, $L_{\mu \nu}$ contains spatial rotations and Lorentz boosts, $D$ is a relativistic dilatation $(z=1)$ and $K_{\mu}$ describes special conformal transformations. For a generator $T$, the dimension $d(T)$ is measured as

$$
[D, T]=d(T) T
$$

It is easy to read the dimensions of the generators

$$
d\left(K_{\mu}, L_{\mu \nu}, D, P_{\mu}\right)=(-1,0,0,1)
$$

from the commutation relations of conformal algebra. The dimensions of the generators in the subset

$$
\left\{P_{\mu}, L_{\mu \nu}, D\right\}
$$

are non-negative and the set (3) forms a subalgebra of (2). Thus we can find out a subalgebra by eliminating negative-dimension generators. This is the case in general and hence should be regarded as a general prescription to pick up a subalgebra, which is known as Borel subalgebra. In fact, this prescription picks up less supersymmetric subalgebra, as we will see later.

Furthermore, a smaller subalgebra of (3) can be found because the dilatation $D$ never appears in the right-hand side of commutators of the generators in (3). Therefore $D$ can be removed and the reduced set of the generators,

$$
\left\{P_{\mu}, L_{\mu \nu}\right\}
$$

forms a subalgebra. This is nothing but the Poincaré algebra. This is also the case in general even if the starting algebra contains many $\mathrm{U}(1)$ charges.

The last is how to introduce an anisotropic scaling generator with an arbitrary $z$. Indeed, the Schrödinger and Lifshitz algebras are obtained from a relativistic conformal algebra by shifting the relativistic dilatation $D(z=1)$ with a certain $\mathrm{U}(1)$ generator $V$ like

$$
D_{z} \equiv D+(z-1) V
$$


The anisotropic dilatation generator $\tilde{D}_{z}$ plays a central role in our discussions.

Furthermore, it would be convenient to introduce two charges $d_{z}$ and $v$ of $D_{z}$ and $V$ defined as, respectively,

$$
\begin{aligned}
& {\left[D_{z}, T\right]=d_{z}(T) T} \\
& {[V, T]=v(T) T}
\end{aligned}
$$

where $T$ is a generator. The $v$ charge enables us to figure out subalgebras pictorially, as we will see later.

\section{Non-Relativistic Superalgebras from $\operatorname{psu}(2,2 \mid 4)$}

We first classify superalgebras with the anisotropic scaling (1) as subalgebras of $\operatorname{psu}(2,2 \mid 4)$, following the prescriptions described in Section 2. The notation and convention of $\operatorname{psu}(2,2 \mid 4)$ are summarized in Appendix A.

Two su(2) subalgebras are contained in $\operatorname{psu}(2,2 \mid 4)$ like

$$
\mathrm{su}(2)+\mathrm{su}(2) \subset \mathrm{su}(2,2) \subset \operatorname{psu}(2,2 \mid 4)
$$

Thus there are two u(1) generators $L^{1}{ }_{1}$ and $\dot{L}^{1}{ }_{1}$, which are the Cartan generators of the two su(2)s. By taking a linear combination of the two generators, a couple of new $\mathrm{u}(1)$ generators are defined as

$$
V \equiv L_{1}^{1}-\dot{L}^{\dot{1}}, \quad U \equiv L_{1}{ }_{1}+\dot{L}^{\dot{1}}{ }_{i}
$$

The $V$ is used to shift $D$ while $U$ generates the two-dimensional space rotation.

The resulting $v$ charges of the generators in $\operatorname{psu}(2,2 \mid 4)$ are summarized as follows:

\begin{tabular}{|c|c|c|c|c|c|}
\hline & $L_{2}^{1}, \dot{L}_{\dot{2}}^{\dot{1}}$ & $Q_{2}^{a}, \dot{Q}_{a \dot{2}}$ & $U, V, D, R_{b}^{a}$ & $Q_{1}^{a}, \dot{Q}_{a \dot{1}}$ & $L^{2}, \dot{L}^{\dot{2}}{ }_{i}$ \\
& $K^{1 \dot{1}}, P_{\dot{2} 2}$ & $S_{a}^{1}, \dot{S}^{a \dot{1}}$ & $K^{1 \dot{2}}, K^{2 \dot{1}}, P_{\dot{1} 2}, P_{\dot{2} 1}$ & $S_{a}^{2}, \dot{S}^{a \dot{2}}$ & $K^{2 \dot{2}}, P_{\dot{1} 1}$ \\
\hline$v$ & -1 & $-1 / 2$ & 0 & $1 / 2$ & 1 \\
\hline
\end{tabular}

The list of $d$ and $v$ charges enables us to represent the generators of $\operatorname{psu}(2,2 \mid 4)$ on the $d-v$ plane as depicted in Figure 1 (For $d$ charges, see Appendix A). This $d-v$ plane picture is very helpful to find out subalgebras. For simplicity, it is convenient to introduce the following notation:

$$
K=K^{1 \dot{1}}, G_{i}=\left(L_{2}^{1}, \dot{L}_{\dot{2}}^{\dot{1}}\right), \quad H=P_{\dot{1} 1}, P_{i}=\left(P_{\dot{1} 2}, P_{\dot{2} 1}\right), \quad M=P_{\dot{2} 2}
$$

Then we shall give some examples of subalgebras of $\operatorname{psu}(2,2 \mid 4)$.

(1) Schrödinger algebra with an arbitrary $z$ and 24 supercharges $(d-v \geq 0)$

To deduce the algebra, we have to use the new dilatation generator $D_{z}$ defined in (4), instead of the relativistic dilatation $D$. Since the generator $V$ appears in the right-hand side of commutators, $V$ must be included when $z \neq 2$. As we explain in detail as the next example, the $z=2$ case is a bit special.

The charges $d_{z}$ and $v$ of the generators are summarized in the following list:

\begin{tabular}{|c|c|c|c|c|c|c|c|c|c|}
\hline & $K$ & $S_{a}^{1}, \dot{S}^{a \dot{1}}$ & $U, R, D_{z}, V$ & $G_{i}$ & $Q_{1}^{a}, \dot{Q}_{a \dot{1}}$ & $Q_{2}^{a}, \dot{Q}_{a \dot{2}}$ & $H$ & $P_{i}$ & $M$ \\
\hline$d_{z}$ & $-z$ & $-z / 2$ & 0 & $1-z$ & $z / 2$ & $(2-z) / 2$ & $z$ & 1 & $2-z$ \\
\hline$v$ & -1 & $-1 / 2$ & 0 & -1 & $1 / 2$ & $-1 / 2$ & 1 & 0 & -1 \\
\hline
\end{tabular}


Figure 1. The $\operatorname{psu}(2,2 \mid 4)$ generators on the $d-v$ plane.

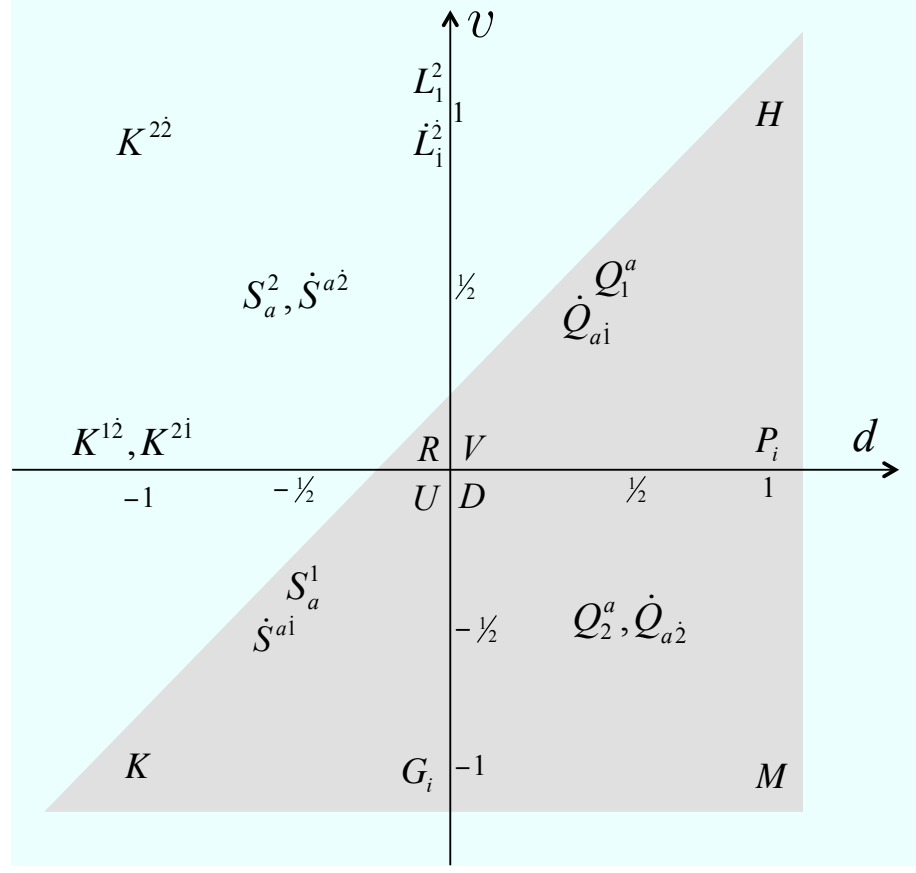

Since $d_{z}$ of time translation generator $H$ is $z$, the dynamical critical exponent also becomes $z$.

The resulting algebra is generated by the set of the charges,

$$
\left\{K, S_{a}^{1}, \dot{S}^{a \dot{1}}, U, R_{b}^{a}, D_{z}, V, G_{i}, Q_{1}^{a}, \dot{Q}_{a \dot{1}}, Q_{2}^{a}, \dot{Q}_{a \dot{2}}, H, P_{i}, M\right\}
$$

The set (8) contains 16 supertranslations and 8 superconformal generators. Hence the amount of supercharges are 24 in total. Note that the generators in (8) are confined in the lower triangular region (shaded) on the $d-v$ plane (not $d_{z}$ but $d !$ ) as depicted in Figure 1. This region is specified by the Schrödinger condition $d-v \geq 0$. Thus the $d-v$ plane picture is very useful to understand a subalgebra pictorially.

The commutation relations of the bosonic part are nothing but those of Schrödinger algebra with an $\operatorname{arbitrary} z$,

$$
\begin{aligned}
& {\left[U, P_{1}\right]=-P_{1}, \quad\left[U, P_{2}\right]=P_{2}} \\
& {\left[H, G_{i}\right]=-P_{i}, \quad\left[G_{i}, P_{j}\right]=\delta_{i j} M, \quad\left[U, G_{1}\right]=-G_{1}, \quad\left[U, G_{2}\right]=G_{2}} \\
& {[K, H]=D_{z}-(z-2) V, \quad\left[K, P_{i}\right]=G_{i}}
\end{aligned}
$$

The (anti-)commutation relations including fermionic generators are

$$
\begin{aligned}
& \left\{Q_{1}^{a}, \dot{Q}_{b \mathrm{i}}\right\}=\delta_{b}^{a} H, \quad\left\{Q_{2}^{a}, \dot{Q}_{b \dot{2}}\right\}=\delta_{b}^{a} M, \quad\left\{Q_{1}^{a}, \dot{Q}_{b \dot{2}}\right\}=\delta_{b}^{a} P_{2}, \quad\left\{\dot{Q}_{a \mathrm{1}}, Q_{2}^{b}\right\}=\delta_{a}^{b} P_{1} \\
& {\left[G_{1}, Q_{1}^{a}\right]=Q_{2}^{a}, \quad\left[G_{2}, \dot{Q}_{a \dot{1}}\right]=\dot{Q}_{a \dot{2}}} \\
& {\left[H, S_{a}^{1}\right]=-\dot{Q}_{a \dot{1}}, \quad\left[H, \dot{S}^{a \dot{1}}\right]=-Q_{1}^{a}, \quad\left[P_{1}, \dot{S}^{a \mathrm{i}}\right]=-Q_{2}^{a}, \quad\left[P_{2}, S_{a}^{1}\right]=-\dot{Q}_{a \dot{2}}} \\
& {\left[K, Q_{1}^{a}\right]=\dot{S}^{a \dot{1}}, \quad\left[K, \dot{Q}_{a \mathrm{i}}\right]=S_{a}^{1}, \quad\left\{S_{a}^{1}, \dot{S}^{b \dot{1}}\right\}=\delta_{a}^{b} K} \\
& \left\{Q_{1}^{a}, S_{b}^{1}\right\}=R_{b}^{a}+\frac{1}{2} \delta_{b}^{a}\left(D_{z}+U-(z-2) V\right), \quad\left\{Q_{2}^{a}, S_{b}^{1}\right\}=\delta_{b}^{a} G_{1}
\end{aligned}
$$




$$
\left\{\dot{Q}_{a \dot{1}}, \dot{S}^{b \dot{1}}\right\}=-R_{a}^{b}+\frac{1}{2} \delta_{a}^{b}\left(D_{z}-U-(z-2) V\right), \quad\left\{\dot{Q}_{a \dot{2}}, \dot{S}^{b \dot{1}}\right\}=\delta_{a}^{b} G_{2}
$$

Here trivial (anti-)commutation relations have been omitted. In addition, $\mathrm{u}(1)^{2}$ and su(4) act on these generators as (5), (6), and (40) in Appendix A.

(2) Schrödinger algebra with $z=2$ and 24 supercharges $(d-v \geq 0)$

As briefly mentioned before, the case with $z=2$ is a bit special because $V$ does not appear in the right-hand side of the (anti-)commutation relations. It implies that the algebra may be closed without $V$.

Anyway, $V$ can be eliminated in this case and thus the reduced algebra is generated by the following set of the generators,

$$
\left\{K, S_{a}^{1}, \dot{S}^{a \mathrm{i}}, U, R_{b}^{a}, D_{2}, G_{i}, Q_{1}^{a}, \dot{Q}_{a \dot{1}}, Q_{2}^{a}, \dot{Q}_{a \dot{2}}, H, P_{i}, M\right\}
$$

The charge $d_{2}$ is assigned as in the following list:

\begin{tabular}{|c|c|c|c|c|c|}
\hline & $K$ & $S_{a}^{1}, \dot{S}^{a \dot{1}}, G_{i}$ & $U, R_{b}^{a}, D_{2}, Q_{2}^{a}, \dot{Q}_{a \dot{2}}, M$ & $Q_{1}^{a}, \dot{Q}_{a \dot{1}}, P_{i}$ & $H$ \\
\hline$d_{2}$ & -2 & -1 & 0 & 1 & 2 \\
\hline
\end{tabular}

This algebra contains 24 supercharges and this is nothing but the super Schrödinger algebra found in $[16,17]$. This super Schrödinger algebra is realized in the $\operatorname{AdS}_{5} \times \mathrm{S}^{5}$ background with a periodic boundary condition for $x^{-}$-direction corresponding to the generator $M$ [18]. The periodic boundary condition breaks 8 superconformal symmetries.

According to the prescription explained in Section 2, it is possible to find out a smaller subalgebra without the dilatation $D_{2}$. This is nothing but a supersymmetric (centrally extended) Galilean algebra.

(3) Schrödinger algebra with an arbitrary $z$ and 16 supercharges $(d-v \geq 0$ and $d \geq 0)$

This algebra is generated by the set of the generators,

$$
\left\{U, R_{b}^{a}, D_{z}, G_{i}, Q_{1}^{a}, \dot{Q}_{a \dot{1}}, Q_{2}^{a}, \dot{Q}_{a \dot{2}}, H, P_{i}, M\right\}
$$

This is the Schrödinger algebra including 16 supercharges. The (anti-)commutation relations are given by (5), (6), (9), (10), (12), (13) and (40). Note that $V$ has been omitted because it does not appear in the right-hand side of the commutators. This is usually referred as the Schrödinger algebra with an arbitrary $z$ in the literature. This subalgebra is specified by imposing an additional condition $d \geq 0$ as well as $d-v \geq 0$. The condition comes from the prescription to eliminate negative dimension generators as explained in Section 2.

Before moving to Lifshitz examples, we would like to comment on gravity solutions preserving super Schrödinger symmetry. Such solutions are reported in many literatures (For example, see [19-26]). Basically, the Schrödinger symmetry is realized by adding a deformation term of pp-wave type to the AdS spacetime. The deformation term breaks the relativistic superconformal algebra to a smaller one like a super Schrödinger algebra discussed here. It is an open problem to construct gravity solutions preserving the super Schrödingier symmetry including $K$ and $V$, because the isometry seems to be 
enhanced to the full relativistic superconformal algebra by adding $K$ and $V$. Less supersymmetric Schrödinger symmetry also appears in this context. All of the symmetry can be found out by considering smaller subalgebras.

(4) Lifshitz algebra with an arbitrary $z$ and 16 supercharges $(d-v \geq 0$ and $d+v \geq 0)$

It is a turn to consider a Lifshitz subalgebra. We consider $D_{z}$ defined in (4). The resulting algebra is generated by the set of the generators,

$$
\left\{U, R_{b}^{a}, D_{z}, Q_{1}^{a}, \dot{Q}_{a \dot{1}}, Q_{2}^{a}, \dot{Q}_{a \dot{2}}, H, P_{i}, M\right\}
$$

Note that $V$ has been omitted because it does not appear in the right-hand side of the commutators. This set contains the usual Lifshitz algebra but there is an additional generator $M$ as the bosonic part, because $M$ is not a center of the algebra any more when $z \neq 2$. The case with $z=2$ is a bit special as we will explain in the next example. It also contains 16 supercharges and thus the resulting algebra should be referred to as the super Lifshitz algebra. This subalgebra can be pictorially understood by imposing an additional Lifshitz condition $d+v \geq 0$ as well as the Schrödinger condition $d-v \geq 0$.

The (anti-)commutation relations are given only by (5), (6), (9), (12) and (40).

(5) Lifshitz algebra with $z=2$ and 16 supercharges $(d-v \geq 0$ and $d+v \geq 0)$

The generator $V$ may be omitted because it does not appear in the right-hand side of the commutators. Then the subalgebra is generated by the set of the generators,

$$
\left\{U, R, D_{2}, Q_{1}^{a}, \dot{Q}_{a \dot{1}}, Q_{2}^{a}, \dot{Q}_{a \dot{2}}, H, P_{i}, M\right\}
$$

The dynamical critical exponent is given by $z=2$ because $d_{2}(H)=2$. The (anti-)commutation relations are given by (5) with $z=2,(6),(9),(12)$ and (40). This is the Lifshitz algebra with 16 supercharges and center $M$. In the bosonic case $M$ does not appear in the right-hand side of commutators and hence it can be eliminated to give the usual Lifshitz algebra. However, in the supersymmetric case, the anti-commutator of $Q_{2}^{a}$ and $\dot{Q}_{a \dot{2}}$ gives rise to $M$. Thus, by restricting to representations with zero central charge $M$, or by dropping $Q_{2}^{a}$ and $\dot{Q}_{a \dot{2}}$, the generator $M$ can also be removed. In the latter case, the resulting 8 super Lifshitz algebra is generated by

$$
\left\{U, R, D_{2}, Q_{1}^{a}, \dot{Q}_{a \dot{1}}, H, P_{i}\right\}
$$

Interestingly, gravity solutions of Lifshitz spacetime preserving 8 super Lifshitz symmetry are found in the literatures [27-33]. The strategy to construct the solutions is the same as in the Schrödinger spacetime.

Note that the original construction of Lifshitz spacetime with $z=2$ starts from a Schrödinger spacetime with $z=0$ [27,28]. This construction can be explained algebraically by identifying the dilatation $D_{0}$ in the Schrödinger spacetime with $z=0$ with the generator $M$ in the Lifshitz spacetime with $z=2$. That is, the Lifshitz algebra with $z=2$ can also be obtained as a subalgebra of Schrödinger algebra with $z=0$. 
Figure 2. The $\operatorname{osp}(8 \mid 4)$ generators on the $d-v$ plane.

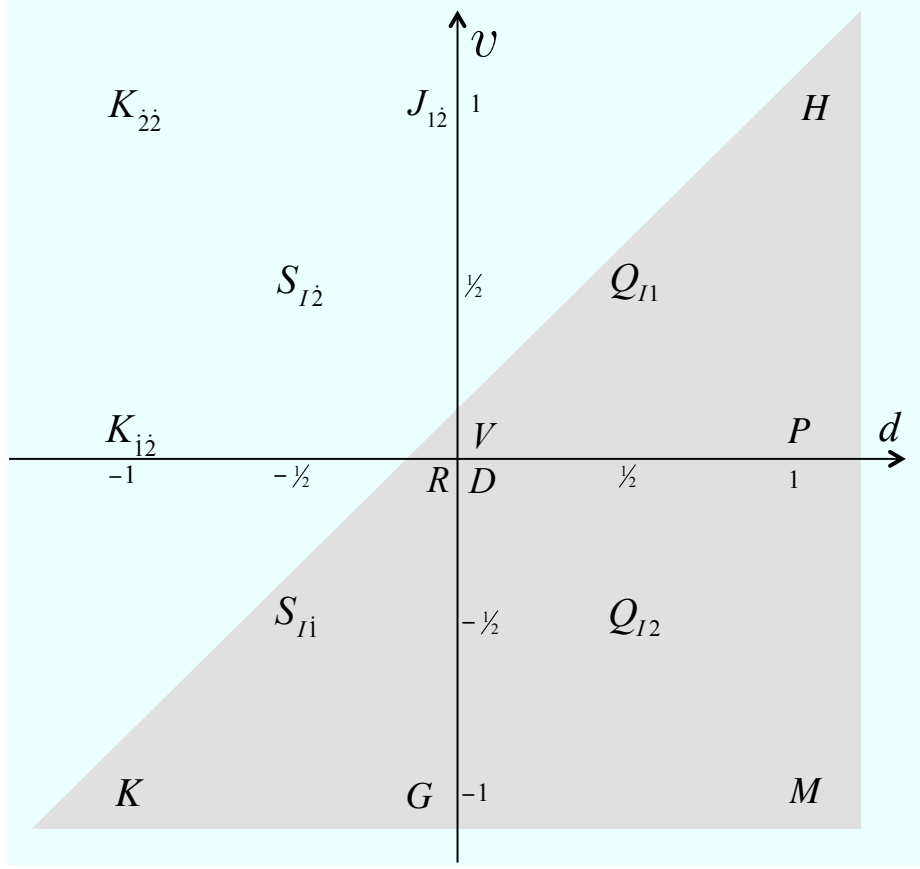

\section{Non-Relativistic Superalgebras from $\operatorname{osp}(8 \mid 4)$}

Next let us consider subalgebras of $\operatorname{sp}(4) \subset \operatorname{osp}(8 \mid 4)$. The detail of our convention and notation for this superalgebra is summarized in Appendix B. The result is basically the same as in the case of $\operatorname{psu}(2,2 \mid 4)$, up to small differences. Thus we will briefly mention the difference and do not try to repeat the same explanation. For example, the relation to gravity solutions is omitted here.

First let us note that $\operatorname{so}(1,2)$ is contained as a subalgebra of $\operatorname{osp}(8 \mid 4)$. Then the diagonal $\mathrm{u}(1)$ generator is contained in so( 1,2$)$ and it is represented by

$$
V \equiv J_{11}
$$

The charge $v(T)$ of a generator $T$ can be measured with (6). The values of $v(T)$ of the generators are summarized in the list:

\begin{tabular}{|c|c|c|c|c|c|}
\hline & $J_{2 \dot{1}}, P_{22}, K_{\mathrm{i1}}$ & $Q_{I 2}, S_{I \dot{1}}$ & $R_{I J}, J_{1 \mathrm{i}}, D, P_{12}, K_{\dot{1} \dot{2}}$ & $Q_{I 1}, S_{I \dot{2}}$ & $J_{1 \dot{2}}, P_{11}, K_{\dot{2} \dot{2}}$ \\
\hline$v$ & -1 & $-1 / 2$ & 0 & $1 / 2$ & 1 \\
\hline
\end{tabular}

For $d$ charges see Appendix B. The generators are expressed on the $d-v$ plane (See Figure 2). For simplicity, it is convenient to introduce the following notation,

$$
K=K_{\mathrm{ii}}, \quad G=J_{2 \mathrm{i}}, \quad H=P_{11}, \quad P=P_{12}, \quad M=P_{22}
$$

As in the case of $\operatorname{psu}(2,2 \mid 4)$, it is possible to find out some subalgebras as follows. 
(1) Schrödinger algebra with an arbitrary $z$ and 24 supercharges $(d-v \geq 0)$

Let us consider the dilatation generator defined in (4). The algebra is generated by the set of the generators,

$$
\left\{K, S_{I \dot{1}}, R_{I J}, D_{z}, V, G, Q_{I 1}, Q_{I 2}, H, P, M\right\}
$$

All of the generators are confined in the lower triangular region (shaded) on the $d-v$ plane as depicted in Figure 2. This region is specified by the Schrödinger condition $d-v \geq 0$ again as in the case of $\operatorname{psu}(2,2 \mid 4)$. The set (20) contains 16 supertranslations and 8 superconformal symmetries. Hence 24 supercharges are included in total. The charges $\tilde{d}_{z}$ and $v$ of the generators are summarized in the list,

\begin{tabular}{|c|c|c|c|c|c|c|c|c|c|}
\hline & $K$ & $S_{I \mathrm{i}}$ & $R_{I J}, D_{z}, V$ & $G$ & $Q_{I 1}$ & $Q_{I 2}$ & $H$ & $P$ & $M$ \\
\hline$d_{z}$ & $-z$ & $-z / 2$ & 0 & $1-z$ & $z / 2$ & $(2-z) / 2$ & $z$ & 1 & $2-z$ \\
\hline$v$ & -1 & $-1 / 2$ & 0 & -1 & $1 / 2$ & $-1 / 2$ & 1 & 0 & -1 \\
\hline
\end{tabular}

It follows that the dynamical critical exponent is $z$ because $d_{z}(H)=z$. The commutation relations of the bosonic subalgebra are given by

$$
\begin{array}{ll}
{[H, G]=-2 P,} & {[P, G]=-M} \\
{[P, K]=-2 G,} & {[H, K]=-4\left(D_{z}-(z-2) V\right)}
\end{array}
$$

The (anti-)commutation relations including fermionic generators are given by

$$
\begin{aligned}
& \left\{Q_{I 1}, Q_{J 1}\right\}=\delta_{I J} H, \quad\left\{Q_{I 1}, Q_{J 2}\right\}=\delta_{I J} P, \quad\left\{Q_{I 2}, Q_{J 2}\right\}=\delta_{I J} M \\
& {\left[G, Q_{I 1}\right]=Q_{I 2}} \\
& {\left[H, S_{I \mathrm{i}}\right]=-2 Q_{I 1}, \quad\left[K, Q_{I 1}\right]=2 S_{I \mathrm{1}}, \quad\left[P, S_{I \mathrm{i}}\right]=-Q_{I 2}} \\
& \left\{Q_{I 1}, S_{J \mathrm{i}}\right\}=\delta_{I J} G, \quad\left\{Q_{I 1}, S_{J 1}\right\}=R_{I J} \delta_{I J}\left(D_{z}-(z-2) V\right)
\end{aligned}
$$

Note that $\mathrm{u}(1)^{2}$ and so(8) act on the generators, following (5), (6), and (47) in Appendix B.

(2) Schrödinger algebra with $z=2$ and 24 super charges $(d-v \geq 0)$

The $z=2$ case is a bit special and the generator $V$ does not appear in the right-hand side of the (anti-)commutation relations. This implies that $V$ may be omitted. After eliminating $V$, the resulting algebra generated by

$$
\left\{K, S_{I \dot{1}}, R_{I J}, D_{2}, G, Q_{I 1}, Q_{I 2}, H, P, M\right\}
$$

is the Schrödinger algebra with 24 supercharges originally found in [16,17]. Note that the bosonic part is rigorously the Schrödinger algebra because $V$ is not contained.

According to the prescription in Section 2, the set (25) contains a supersymmetric (centrally extended) Galilean algebra as a subalgebra. 
(3) Schrödinger algebra with an arbitrary $z$ and 16 supercharges $(d-v \geq 0$ and $d \geq 0)$

This algebra is generated by the set of the generators,

$$
\left\{R_{I J}, D_{z}, G, Q_{I 1}, Q_{I 2}, H, P, M\right\}
$$

The region in the $d-v$ plane where all of the generator are confined is specified by an additional condition $d \geq 0$ as well as the Schrödinger condition $d-v \geq 0$. This set contains 16 supercharges (only supertranslations). The generator $V$ has been omitted because it does not appear in the right-hand side of the commutators. The (anti-)commutation relations are given by (5), (6), (21), (23), (24) and (47). The generator $M$ is now a center.

(4) Lifshitz algebra with an arbitrary $z$ and 16 supercharges $(d-v \geq 0$ and $d+v \geq 0)$

In the same way as in the case of psu(2,2|4), it is possible to find out a super Lifshitz algebra. This algebra is generated by the set of the generators,

$$
\left\{R_{I J}, D_{z}, Q_{I 1}, Q_{I 2}, H, P, M\right\}
$$

All of the generators are confined the region specified by the Lifshitz condition $d+v \geq 0$ as well as the Schrödinger condition $d-v \geq 0$. The generator $V$ has been omitted again because it does not appear in the right-hand side of the commutators. The (anti-)commutation relations are given by (5), (6), (23) and (47) .

In a special case with $z=2$, the generator $M$ becomes a center but it appears in the anti-commutator of $Q_{I 2}$ 's. However, by restricting to representations with zero central charge $M$, or by removing supercharges $Q_{I 2}$, the exact Lifshitz algebra is reproduced.

\section{Non-Relativistic Superalgebras from $\operatorname{osp}\left(8^{*} \mid 4\right)$}

Finally we consider the case of $\operatorname{osp}\left(8^{*} \mid 4\right)$. The notation and convention for $\operatorname{osp}\left(8^{*} \mid 4\right)$ are summarized in Appendix C. The result is basically the same as in the case of $\operatorname{psu}(2,2 \mid 4)$ and $\operatorname{osp}(8 \mid 4)$ again, up to notational differences. We will briefly mention the differences as in the previous section.

Let us consider the diagonal $\mathrm{u}(1)$ generator defined as

$$
V \equiv-J_{1 \dot{4}}+J_{4 \dot{1}}=-J_{2 \dot{3}}+J_{3 \dot{2}}
$$

which is contained in $\operatorname{so}(1,5) \subset$ so* $^{*}(8)$. Then the $v$ charge is assigned to the generators as in the list:

\begin{tabular}{|c|c|c|c|c|c|}
\hline & $K_{\dot{1} \dot{4}}, J_{2 \dot{1}}, J_{3 \dot{1}}$, & $Q_{2 A}, Q_{3 A}$ & $L_{A B}, U^{(1)}$ & $Q_{1 A}, Q_{4 A}$ & $K_{\dot{2} \dot{3}}, J_{1 \dot{2}}, J_{1 \dot{3}}$, \\
& $P_{23}, J_{2 \dot{4}}, J_{3 \dot{4}}$ & $S_{\dot{1} A}, S_{\dot{4} A}$, & $D, V, U^{(2)}$ & $S_{\dot{2} A}, S_{\dot{3} A}$, & $P_{14}, J_{4 \dot{2}}, J_{4 \dot{3}}$ \\
\hline$v$ & -1 & $-1 / 2$ & 0 & $1 / 2$ & 1 \\
\hline
\end{tabular}

Here we have defined

$$
\begin{aligned}
U^{(1)} & \equiv\left\{U_{0}^{(1)}=\frac{1}{2}\left(J_{1 \dot{4}}+J_{4 \dot{1}}\right), U_{-}^{(1)}=J_{1 \dot{1}}, U_{+}^{(1)}=J_{4 \dot{4}}\right\} \\
U^{(2)} & \equiv\left\{U_{0}^{(2)}=\frac{1}{2}\left(J_{2 \dot{3}}+J_{3 \dot{2}}\right), U_{+}^{(2)}=J_{2 \dot{2}}, U_{-}^{(2)}=J_{3 \dot{3}}\right\}
\end{aligned}
$$


Figure 3. The $\operatorname{osp}\left(8^{*} \mid 4\right)$ generators on the $d-v$ plane.

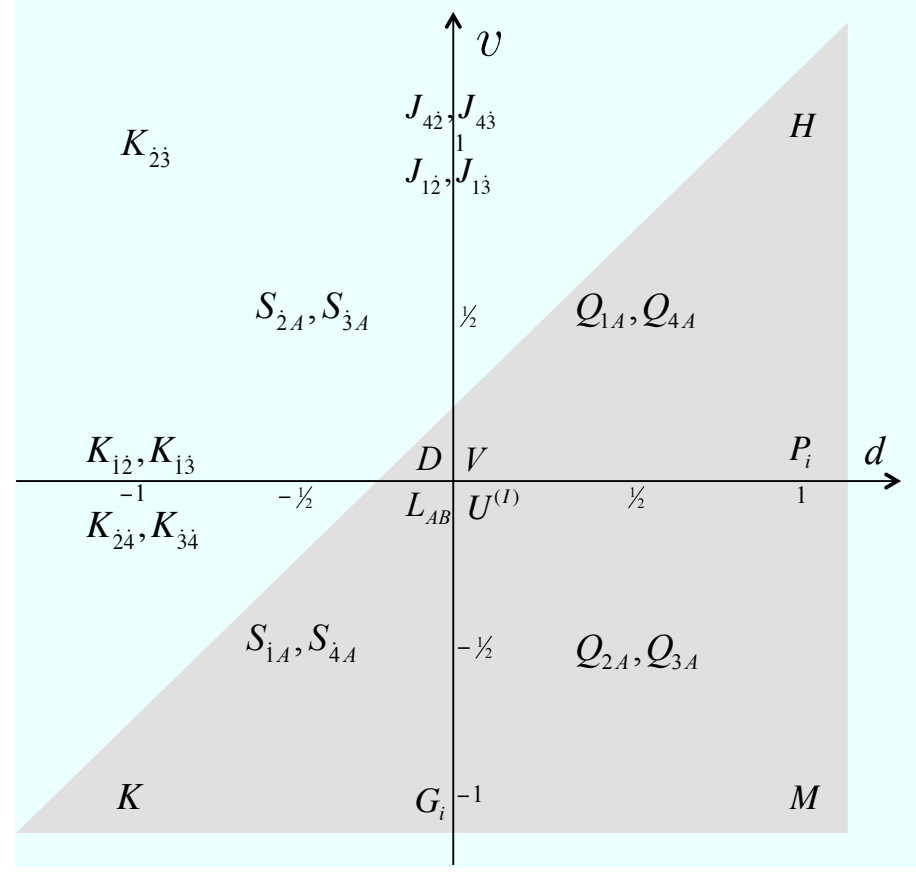

The $U^{(I)}$ with $I=1,2$ generate two su(2)'s , respectively,

$$
\left[U_{0}^{(I)}, U_{ \pm}^{(I)}\right]= \pm U_{ \pm}^{(I)}, \quad\left[U_{+}^{(I)}, U_{-}^{(I)}\right]=-2 U_{0}^{(I)}
$$

This implies that $\left\{U^{(1)}, U^{(2)}\right\}$ generates an so(4) symmetry after all.

The generators are represented on the $d-v$ plane as depicted in Figure 3 (For $d$ charges see Appendix C). For simplicity, it is convenient to introduce the following notation,

$$
\begin{array}{ll}
K=K_{1 \dot{4}}, & G_{i}=\left(-J_{2 \dot{1}},-J_{3 \dot{1}}, J_{2 \dot{4}}, J_{3 \dot{4}}\right) \\
H=P_{14}, & P_{i}=\left(P_{12}, P_{13}, P_{24}, P_{34}\right), \quad M=P_{23}
\end{array}
$$

Let see some examples of subalgebras of $\operatorname{osp}\left(8^{*} \mid 4\right)$ below.

(1) Schrödinger algebra with an arbitrary $z$ and 24 supercharges $(d-v \geq 0)$

We use the dilatation generator $D_{z}$ defined in (4). The algebra is generated by the set of the generators,

$$
\left\{K, S_{\dot{1} A}, S_{\dot{4} A}, L_{A B}, D_{z}, V, U^{(1)}, U^{(2)}, G_{i}, Q_{a A}, H, P_{i}, M\right\}
$$

All of the generators are confined in the lower triangular region (shaded) on the $d-v$ plane as depicted in Figure 3. This region is specified by the Schrödinger condition $d-v \geq 0$. The set (29) contains 16 supertranslations and 8 superconformal symmetries. Thus the total amount of supercharges is 24 . The charges $d_{z}$ and $v$ are summarized in the list:

\begin{tabular}{|c|c|c|c|c|c|c|c|c|c|}
\hline & $K$ & $S_{1 A}, S_{\dot{4} A}$ & $L_{A B}, D_{z}, V, U^{(1)}, U^{(2)}$ & $G_{i}$ & $Q_{1 A}, Q_{4 A}$ & $Q_{2 A}, Q_{3 A}$ & $H$ & $P_{i}$ & $M$ \\
\hline$d_{z}$ & $-z$ & $-z / 2$ & 0 & $1-z$ & $z / 2$ & $(2-z) / 2$ & $z$ & 1 & $2-z$ \\
\hline$v$ & -1 & $-1 / 2$ & 0 & -1 & $1 / 2$ & $-1 / 2$ & 1 & 0 & -1 \\
\hline
\end{tabular}


It follows that the dynamical critical exponent is $z$ because $d_{z}(H)=z$. The commutation relations of bosonic subalgebra are

$$
\begin{array}{ll}
{\left[H, G_{i}\right]=P_{i},} & {\left[P_{i}, G_{j}\right]=\alpha_{i j} M} \\
{\left[P_{i}, K\right]=G_{i},} & {[H, K]=-D_{z}+(z-2) V}
\end{array}
$$

Here $\alpha_{i j}$ are defined as

$$
\alpha_{14}=\alpha_{41}=1, \quad \alpha_{23}=\alpha_{32}=-1, \quad \text { others }=0
$$

The (anti-)commutation relations including fermionic generators are

$$
\begin{aligned}
& \left\{Q_{1 A}, Q_{4 A}\right\}=-\mathcal{J}_{A B} H, \quad\left\{Q_{1 A}, Q_{2 A}\right\}=-\mathcal{J}_{A B} P_{1}, \quad\left\{Q_{1 A}, Q_{3 A}\right\}=-\mathcal{J}_{A B} P_{2} \\
& \left\{Q_{2 A}, Q_{3 A}\right\}=-\mathcal{J}_{A B} M, \quad\left\{Q_{2 A}, Q_{4 A}\right\}=-\mathcal{J}_{A B} P_{3}, \quad\left\{Q_{3 A}, Q_{4 A}\right\}=-\mathcal{J}_{A B} P_{4} \\
& {\left[G_{1}, Q_{4 A}\right]=-Q_{2 A}, \quad\left[G_{2}, Q_{4 A}\right]=-Q_{3 A}, \quad\left[G_{3}, Q_{1 A}\right]=-Q_{2 A}, \quad\left[G_{4}, Q_{1 A}\right]=-Q_{3 A}} \\
& {\left[H, S_{\dot{1} A}\right]=Q_{1 A}, \quad\left[H, S_{\dot{4} A}\right]=Q_{4 A}, \quad\left[K, Q_{1 A}\right]=-S_{\dot{1} A}, \quad\left[K, Q_{4 A}\right]=-S_{\dot{4} A}} \\
& {\left[P_{1}, S_{\dot{4} A}\right]=Q_{2 A}, \quad\left[P_{2}, S_{\dot{4} A}\right]=Q_{3 A}, \quad\left[P_{3}, S_{\dot{1 A}_{A}}\right]=Q_{2 A}, \quad\left[P_{4}, S_{\dot{1 A}_{A}}\right]=Q_{3 A}} \\
& \left\{Q_{2 A}, S_{\dot{1 B}}\right\}=\mathcal{J}_{A B} G_{1}, \quad\left\{Q_{3 A}, S_{\dot{1}}\right\}=\mathcal{J}_{A B} G_{2}, \quad\left\{Q_{2 A}, S_{\dot{4} B}\right\}=\mathcal{J}_{A B} G_{3} \\
& \left\{Q_{3 A}, S_{\dot{4} B}\right\}=\mathcal{J}_{A B} G_{4}, \quad\left\{S_{\dot{1} A}, S_{\dot{4} B}\right\}=-\mathcal{J}_{A B} K, \quad\left\{Q_{1 A}, S_{\dot{1} B}\right\}=-\mathcal{J}_{A B} U_{-}^{(1)} \\
& \left\{Q_{1 A}, S_{\dot{4} B}\right\}=-L_{A B}+\frac{1}{2} \mathcal{J}_{A B}\left(D_{z}-2 U_{0}^{(1)}+(2-z) V\right), \quad\left\{Q_{4 A}, S_{\dot{4} B}\right\}=-\mathcal{J}_{A B} U_{+}^{(1)} \\
& \left\{Q_{4 A}, S_{1 B}\right\}=L_{A B}-\frac{1}{2} \mathcal{J}_{A B}\left(D_{z}+2 U_{0}^{(1)}+(2-z) V\right)
\end{aligned}
$$

Note that the bosonic symmetry generators of $\mathrm{u}(1)^{2}$, so(4) and so(5) act on the generators in the obvious way, following (5), (6), (28), and (49) in Appendix C, respectively.

(2) Schrödinger algebra with $z=2$ and 24 supercharges $(d-v \geq 0)$

The case with $z=2$ is a bit special again. Then $V$ does not appear in the right-hand side of commutators. It implies that $V$ may be eliminated when $z=2$. The reduced algebra is generated by the set of the generators,

$$
\left\{K, S_{\dot{1} A}, S_{\dot{4} A}, L_{A B}, D_{2}, U^{(1)}, U^{(2)}, G_{i}, Q_{a A}, H, P_{i}, M\right\}
$$

It contains 24 supercharges in total and is the same as the result found in [16,17]. A supersymmetric (centrally extended) Galilean algebra can also be found as a subalgebra.

(3) Schrödinger algebra with an arbitrary $z$ and 16 supercharges $(d-v \geq 0$ and $d \geq 0)$

The algebra is generated by the set of the generators,

$$
\left\{L_{A B}, D_{z}, U^{(1)}, U^{(2)}, G_{i}, Q_{a A}, H, P_{i}, M\right\}
$$

The generators are confined in the region specified by an additional condition $d \geq 0$ as well as the Schrödinger condition $d-v \geq 0$. It contains 16 supercharges. The generator $V$ has been omitted because it does not appear in the right-hand side of the commutators. The (anti-)commutation relations are given by (5), (6), (30), (32), (33), (28) and (49). 
(4) Lifshitz algebra with an arbitrary $z$ and 16 supercharges $(d-v \geq 0$ and $d+v \geq 0)$

This algebra is generated by the set of the generators,

$$
\left\{L_{A B}, D_{z}, U^{(1)}, U^{(2)}, Q_{a A}, H, P_{i}, M\right\}
$$

The generators are in the region fixed by the Lifshitz condition $d+v \geq 0$ and the Schrödinger condition $d-v \geq 0$. This set contains 16 supersymmetries. Again we have omitted $V$ because it does not appear in the right-hand side of the commutators. The (anti-)commutation relations are given by (5), (6), (28), (32) and (49).

In the same way as in the case of $\operatorname{psu}(2,2 \mid 4)$ and $\operatorname{osp}(8 \mid 4)$, it is possible to realize the exact Lifshitz algebra when $z=2$, by restricting to representations with zero central charge $M$ or by removing half of supersymmetries.

\section{Summary}

We have presented a classification of superalgebras with the anisotropic scaling (1) as subalgebras of the superalgebras: $\operatorname{psu}(2,2 \mid 4), \operatorname{osp}(8 \mid 4)$ and osp $\left(8^{*} \mid 4\right)$, which are concerned with AdS/CFT in type IIB string and $\mathrm{M}$ theories. Our method to extract subalgebras is basically to find Borel subalgebras of these superalgebras. It enables us to derive non-relativistic scaling algebras systematically. In particular, we have considered two $\mathrm{u}(1)$ charges, $d$ and $v$, and supersymmetric extensions of Schrödinger algebra and Lifshitz algebra have been obtained. This method provides us a pictorial understanding on non-relativistic scaling superalgebras obtained in the previous works [16,17]. Furthermore, we have extracted Schrödinger superalgebras and Lifshitz superalgebras with arbitrary $z$. We hope that our result would be useful in constructing gravity solutions preserving superalgebras including the anisotropic scaling and in discussing the relation to the field-theory side.

\section{Acknowledgments}

This work was supported by the scientific grants from the ministry of education, science, sports, and culture of Japan (No. 22740160), and in part by the Grant-in-Aid for the Global COE Program "The Next Generation of Physics, Spun from Universality and Emergence" from the Ministry of Education, Culture, Sports, Science and Technology (MEXT) of Japan.

\section{A. $\quad \operatorname{psu}(2,2 \mid 4)$}

Let us introduce the superalgebra, psu(2,2|4), following the notation of [34]. The number in a box represents the number of independent generators.

We begin with $\mathrm{u}(2,2 \mid 4)$, in which the (anti-)commutation relations are given by

- $\operatorname{su}(2) \quad L^{\alpha}{ }_{\beta}\left(L^{1}{ }_{1}=-L_{2}^{2}, L^{1}{ }_{2}\right.$ and $\left.L^{2}{ }_{1}\right) \quad 3$

$$
\left[L^{\alpha}{ }_{\beta}, J_{\gamma}\right]=\delta_{\gamma}^{\alpha} J_{\beta}-\frac{1}{2} \delta_{\beta}^{\alpha} J_{\gamma}, \quad\left[L^{\alpha}{ }_{\beta}, J^{\gamma}\right]=-\delta_{\beta}^{\gamma} J^{\alpha}+\frac{1}{2} \delta_{\beta}^{\alpha} J^{\gamma}
$$


- $\operatorname{su}(2) \quad \dot{L}^{\dot{\alpha}}{ }_{\dot{\beta}}\left(\dot{L}^{\dot{1}}{ }_{i}=-\dot{L}^{\dot{2}}{ }_{2}, \dot{L}_{\dot{2}}^{\dot{1}}\right.$ and $\left.\dot{L}^{\dot{2}}{ }_{1}\right)$

$$
\left[\dot{L}^{\dot{\alpha}}{ }_{\dot{\beta}}, J_{\dot{\gamma}}\right]=\delta_{\dot{\gamma}}^{\dot{\alpha}} J_{\dot{\beta}}-\frac{1}{2} \delta_{\dot{\beta}}^{\dot{\alpha}} J_{\dot{\gamma}}, \quad\left[\dot{L}_{\dot{\beta}}^{\dot{\alpha}}, J^{\dot{\gamma}}\right]=-\delta_{\dot{\beta}}^{\dot{\gamma}} J^{\dot{\alpha}}+\frac{1}{2} \delta_{\dot{\beta}}^{\dot{\alpha}} J^{\dot{\gamma}}
$$

- $\operatorname{su}(4) R_{b}^{a}(a, b, c=1,2,3,4)$

$$
\left[R_{b}^{a}, J_{c}\right]=\delta_{c}^{a} J_{b}-\frac{1}{4} \delta_{b}^{a} J_{c}, \quad\left[R_{b}^{a}, J^{c}\right]=-\delta_{b}^{c} J^{a}+\frac{1}{4} \delta_{b}^{a} J^{c}
$$

- dilatation $D$

$$
[D, J]=d(J) J
$$

\begin{tabular}{|c|c|c|c|c|c|}
\hline & $K$ & $S, \dot{S}$ & $L, R$ & $Q, \dot{Q}$ & $P$ \\
\hline$d$ & -1 & $-1 / 2$ & 0 & $1 / 2$ & 1 \\
\hline
\end{tabular}

- hypercharge $B$

$$
[B, J]=\operatorname{hyp}(J) J, \quad \operatorname{hyp}(Q, \dot{Q}, S, \dot{S})=\left(\frac{1}{2},-\frac{1}{2},-\frac{1}{2}, \frac{1}{2}\right)
$$

- central charge $C$

- supertranslation $Q_{\beta}^{a} \quad$ (its conjugate $\dot{Q}_{\dot{\alpha} b}$ ) superconformal symmetry $S_{b}^{\alpha} \quad$ (its conjugate $\dot{S}^{a \dot{\beta}}$ ) $16+16$

$$
\begin{aligned}
& \left\{\dot{Q}_{\dot{\alpha} a}, Q_{\beta}^{b}\right\}=\delta_{a}^{b} P_{\dot{\alpha} \beta} \quad\left\{\dot{S}^{a \dot{\alpha}}, S_{b}^{\beta}\right\}=\delta_{b}^{a} K^{\beta \dot{\alpha}} \\
& \left\{S_{a}^{\alpha}, Q_{\beta}^{b}\right\}=\delta_{a}^{b} L^{\alpha}{ }_{\beta}+\delta_{\beta}^{\alpha} R_{a}^{b}+\frac{1}{2} \delta_{a}^{b} \delta_{\beta}^{\alpha}(D-C) \\
& \left\{\dot{S}^{a \dot{\alpha}}, \dot{Q}_{\dot{\beta} b}\right\}=\delta_{b}^{a} \dot{L}_{\dot{\beta}}^{\dot{\alpha}}-\delta_{\dot{\beta}}^{\dot{\alpha}} R^{a}{ }_{b}+\frac{1}{2} \delta_{b}^{a} \delta_{\dot{\beta}}^{\dot{\alpha}}(D+C)
\end{aligned}
$$

- translation $P_{\dot{\alpha} \beta}$ and special conformal transformation $K^{\alpha \dot{\beta}} \quad 4+4$

$$
\begin{aligned}
& {\left[S_{a}^{\alpha}, P_{\dot{\beta} \gamma}\right]=\delta_{\gamma}^{\alpha} \dot{Q}_{\dot{\beta} a} \quad\left[\dot{S}^{a \dot{\alpha}}, P_{\dot{\beta} \gamma}\right]=\delta_{\dot{\beta}}^{\dot{\alpha}} Q_{\gamma}^{a}} \\
& {\left[K^{\alpha \dot{\beta}}, \dot{Q}_{\dot{\gamma} c}\right]=\delta_{\dot{\gamma}}^{\dot{\beta}} S_{c}^{\alpha} \quad\left[K^{\alpha \dot{\beta}}, Q_{\gamma}^{c}\right]=\delta_{\gamma}^{\alpha} \dot{S}^{c \dot{\beta}}} \\
& {\left[K^{\alpha \dot{\beta}}, P_{\dot{\gamma} \delta}\right]=\delta_{\dot{\gamma}}^{\dot{\beta}} L^{\alpha}{ }_{\delta}+\delta_{\delta}^{\alpha} \dot{L}^{\dot{\beta}} \dot{\gamma}+\delta_{\delta}^{\alpha} \delta_{\dot{\gamma}}^{\dot{\beta}} D}
\end{aligned}
$$

We are interested in $\operatorname{psu}(2,2 \mid 4)$ and so we set $B=C=0$ here.

B. $\quad \operatorname{osp}(p \mid 2 q)$

A superalgebra is a $\mathbb{Z}_{2}$-graded linear space $g=g^{0} \oplus g^{1}$ with multiplication, Lie superbracket [ , \}: $g \times g \rightarrow g$ characterized by the three properties

$$
\begin{aligned}
& {[a, b\}=-(-1)^{|a||b|}[b, a\}} \\
& |[a, b\}|=|a|+|b| \bmod 2 \\
& {[[a, b\}, c\}-(-1)^{|c|(|a|+|b|)}[[c, a\}, b\}-(-1)^{|a|(|b|+|c|)}[[b, c\}, a\}=0}
\end{aligned}
$$


where

$$
|a|= \begin{cases}1 & \text { if } a \in g^{1} \\ 0 & \text { if } a \in g^{0}\end{cases}
$$

It follows from (1.2) that

$$
\left[g^{0}, g^{0}\right] \subset g^{0}, \quad\left[g^{0}, g^{1}\right] \subset g^{1}, \quad\left\{g^{1}, g^{1}\right\} \subset g^{0}
$$

The elements in $g^{0}\left(g^{1}\right)$ are called even (odd).

Let us consider the set of all $(p+2 q) \times(p+2 q)$ complex matrices

$$
F=\left(\begin{array}{cc}
A_{p \times p} & i B_{p \times 2 q} \\
C_{2 q \times p} & D_{2 q \times 2 q}
\end{array}\right)
$$

which forms a superalgebra $\operatorname{gl}(p \mid 2 q ; \mathbb{C})$. By imposing the condition that the supertrace should vanish,

$$
\operatorname{str} F \equiv \operatorname{tr} A-\operatorname{tr} D \equiv 0
$$

the superalgebra reduces to $\operatorname{sl}(p \mid 2 q ; \mathbb{C})$. The superalgebra $\operatorname{osp}(p \mid 2 q ; \mathbb{C})$ is a subalgebra of $\operatorname{sl}(p \mid 2 q ; \mathbb{C})$ under an additional condition

$$
F^{s T} \Omega+\Omega F=0
$$

Here we have introduced the following quantities,

$$
F^{s T}=\left(\begin{array}{cc}
A^{T} & -C^{T} \\
i B^{T} & D^{T}
\end{array}\right) \quad \Omega \equiv\left(\begin{array}{cc}
1_{p \times p} & 0 \\
0 & i \mathcal{J}_{2 q \times 2 q}
\end{array}\right), \quad \mathcal{J} \equiv\left(\begin{array}{cc}
0 & 1_{q \times q} \\
-1_{q \times q} & 0
\end{array}\right)
$$

Namely,

$$
A^{T}=-A, \quad B=C^{T} \mathcal{J}, \quad D^{T} \mathcal{J}+\mathcal{J} D=0
$$

which imply that $A \in \operatorname{so}(p)$ and $D \in \mathrm{sp}(2 q)$. Furthermore, one may impose the reality condition

$$
F^{s *}=F, \quad F^{s *}=\left(\begin{array}{cc}
A^{*} & i B^{*} \\
C^{*} & D^{*}
\end{array}\right)
$$

This is compatible with (43) and then the superalgebra is $\operatorname{osp}(p \mid 2 q ; \mathbb{R})$ with $A^{*}=A, B^{*}=B, C^{*}=C$ and $D^{*}=D$.

It is convenient to consider the following matrix,

$$
M=F \Omega=\left(\begin{array}{cc}
A & -B \mathcal{J} \\
C & i D \mathcal{J}
\end{array}\right)
$$

where $F \in \operatorname{osp}(p \mid 2 q)$. It follows that

$$
A^{T}=-A, \quad C^{T}=-B \mathcal{J}, \quad(D \mathcal{J})^{T}=D \mathcal{J}
$$


Let us denote the components of $M$ like

$$
M_{I J}=R_{I J}=-R_{J I}, \quad M_{A B}=i L_{A B}=i L_{B A}, \quad M_{I A}=Q_{I A}=Q_{A I}
$$

where $I, J=1, \cdots, p$ and $A=1, \cdots, 2 q$. Then the (anti-)commutation relations of $o s p(p \mid 2 q)$ are

$$
\begin{aligned}
{\left[R_{I J}, R_{K L}\right] } & =\delta_{J K} R_{I L}-\delta_{J L} R_{I K}-\delta_{I K} R_{J L}+\delta_{I L} R_{J K} \\
{\left[L_{A B}, L_{C D}\right] } & =-\mathcal{J}_{B C} L_{A D}-\mathcal{J}_{B D} L_{A C}-\mathcal{J}_{A C} L_{B D}-\mathcal{J}_{A D} L_{B C} \\
{\left[R_{I J}, Q_{K A}\right] } & =\delta_{J K} Q_{I A}-\delta_{I K} Q_{J A} \\
{\left[L_{A B}, Q_{I C}\right] } & =-\mathcal{J}_{B C} Q_{I A}-\mathcal{J}_{A C} Q_{I B} \\
\left\{Q_{I A}, Q_{J B}\right\} & =\delta_{I J} L_{A B}-\mathcal{J}_{A B} R_{I J}
\end{aligned}
$$

Example: $\operatorname{osp}(8 \mid 4)$

As a concrete example, let us consider osp(8|4). Its (anti-)commutation relations are given by

$$
\begin{aligned}
{\left[R_{I J}, R_{K L}\right] } & =\delta_{J K} R_{I L}-\delta_{J L} R_{I K}-\delta_{I K} R_{J L}+\delta_{I L} R_{J K} \\
{\left[L_{A B}, L_{C D}\right] } & =-\mathcal{J}_{B C} L_{A D}-\mathcal{J}_{B D} L_{A C}-\mathcal{J}_{A C} L_{B D}-\mathcal{J}_{A D} L_{B C} \\
{\left[R_{I J}, Q_{K A}\right] } & =\delta_{J K} Q_{I A}-\delta_{I K} Q_{J A} \\
{\left[L_{A B}, Q_{I C}\right] } & =-\mathcal{J}_{B C} Q_{I A}-\mathcal{J}_{A C} Q_{I B} \\
\left\{Q_{I A}, Q_{J B}\right\} & =\delta_{I J} L_{A B}-\mathcal{J}_{A B} R_{I J}
\end{aligned}
$$

where

- $R_{I J}=-R_{J I}(I, J=1, \cdots, 8)$ generates $\operatorname{so}(8) \quad 28$,

- $L_{A B}=L_{B A}(A=1, \cdots, 4)$ generates $\operatorname{sp}(4) \quad 10$, and $\mathcal{J}_{A B}$ is the symplectic form .

Let us decompose $A$ into $(\alpha, \dot{\alpha})$ with $\alpha, \dot{\alpha}=1,2$. Then the related quantities are rewritten as

$$
L_{A B}=\left(\begin{array}{cc}
P_{\alpha \beta} & J_{\alpha \dot{\beta}}+\frac{2}{q} D \delta_{\alpha \dot{\beta}} \\
J_{\dot{\alpha} \beta}+\frac{2}{q} D \delta_{\dot{\alpha} \beta} & K_{\dot{\alpha} \dot{\beta}}
\end{array}\right), \quad Q_{I A}=\left(Q_{I \alpha}, Q_{I \dot{\alpha}}\right)
$$

where we take $q=2$ for $\operatorname{osp}(8 \mid 4)$ and $\delta_{\alpha \dot{\beta}}=\delta_{\alpha \beta}$ Note that

$$
\operatorname{tr} J_{a \dot{\beta}}=J_{1 \dot{1}}+J_{2 \dot{2}}=0
$$

and hence $J_{11}, J_{12}$ and $J_{2 i}$ are regarded as independent generators. It is straightforward to rewrite the (anti-)commutation relations of $\operatorname{osp}(8 \mid 4)$ in terms of these generators.

- $\operatorname{so}(8)$

$$
\left[R_{I J}, O_{K}\right]=\delta_{J K} O_{I}-\delta_{I K} O_{J}
$$

- $\operatorname{so}(1,2) \quad\left(J_{1 \dot{1}}=-J_{2 \dot{2}}, J_{1 \dot{2}}\right.$ and $\left.J_{2 \dot{1}}\right)$

$$
\left[J_{\alpha \dot{\beta}}, O_{\gamma}\right]=\delta_{\dot{\beta} \gamma} O_{\alpha}-\frac{1}{q} \delta_{\alpha \dot{\beta}} O_{\gamma}, \quad\left[J_{\alpha \dot{\beta}}, O_{\dot{\gamma}}\right]=-\delta_{\alpha \dot{\gamma}} O_{\dot{\beta}}+\frac{1}{q} \delta_{\alpha \dot{\beta}} O_{\dot{\gamma}}
$$


- dilatation

$$
\begin{gathered}
1 \\
{[D, O]=d(O) O}
\end{gathered}
$$

\begin{tabular}{|c|c|c|c|c|c|}
\hline & $K_{\dot{\alpha} \dot{\beta}}$ & $S_{I \dot{\alpha}}$ & $J_{\alpha \dot{\beta}}, D, R_{I J}$ & $Q_{I \alpha}$ & $P_{\alpha \beta}$ \\
\hline$d$ & -1 & $-1 / 2$ & 0 & $1 / 2$ & 1 \\
\hline
\end{tabular}

- supertranslation $Q_{I \alpha}$ and superconformal symmetry $S_{I \dot{\alpha}} \quad 16+16$

$$
\begin{aligned}
\left\{Q_{I \alpha}, Q_{J \beta}\right\} & =\delta_{I J} P_{\alpha \beta}, \quad\left\{S_{I \dot{\alpha}}, S_{J \dot{\beta}}\right\}=\delta_{I J} K_{\dot{\alpha} \dot{\beta}} \\
\left\{Q_{I \alpha}, S_{J \dot{\beta}}\right\} & =\delta_{I J} J_{\alpha \dot{\beta}}+\delta_{\alpha \dot{\beta}}\left(\frac{2}{q} \delta_{I J} D-R_{I J}\right)
\end{aligned}
$$

- translation $P_{\alpha \beta}\left(=P_{\beta \alpha}\right)$ and special conformal transformation $K_{\dot{\alpha} \dot{\beta}}\left(=K_{\dot{\beta} \dot{\alpha}}\right)$

$$
\begin{aligned}
& {\left[P_{\alpha \beta}, S_{I \dot{\gamma}}\right]=-\delta_{\beta \dot{\gamma}} Q_{I \alpha}-\delta_{\alpha \dot{\gamma}} Q_{I \beta}, \quad\left[K_{\dot{\alpha} \dot{\beta}}, Q_{I \gamma}\right]=\delta_{\dot{\beta} \gamma} S_{I \dot{\alpha}}+\delta_{\dot{\alpha} \gamma} S_{I \dot{\beta}}} \\
& {\left[P_{\alpha \beta}, K_{\dot{\gamma} \dot{\delta}}\right]=-\delta_{\beta \dot{\gamma}} J_{\alpha \dot{\delta}}-\delta_{\alpha \dot{\gamma}} J_{\beta \dot{\delta}}-\delta_{\beta \dot{\delta}} J_{\alpha \dot{\gamma}}-\delta_{\alpha \dot{\delta}} J_{\beta \dot{\gamma}}-\frac{4}{q}\left(\delta_{\beta \dot{\gamma}} \delta_{\alpha \dot{\delta}}+\delta_{\alpha \dot{\gamma}} \delta_{\beta \dot{\delta}}\right) D}
\end{aligned}
$$

\section{C. $\operatorname{osp}\left(8^{*} \mid 4\right)$}

We introduce here the superalgebra, $\operatorname{osp}\left(8^{*} \mid 4\right)$. Its bosonic part is

$$
\mathrm{so}^{*}(8) \oplus \mathrm{usp}(4) \simeq \mathrm{so}(2,6) \oplus \mathrm{so}(5) \quad 28+10
$$

It is convenient to work with matrices with spinor indices for so(2,6) below [35]. The (anti-)commutation relations of $\operatorname{osp}\left(8^{*} \mid 4\right)$ are given by

$$
\begin{aligned}
{\left[R_{I J}, R_{K L}\right] } & =C_{J K} R_{I L}-C_{J L} R_{I K}-C_{I K} R_{J L}+C_{I L} R_{J K} \\
{\left[L_{A B}, L_{C D}\right] } & =-\mathcal{J}_{B C} L_{A D}-\mathcal{J}_{B D} L_{A C}-\mathcal{J}_{A C} L_{B D}-\mathcal{J}_{A D} L_{B C} \\
{\left[R_{I J}, Q_{K A}\right] } & =C_{J K} Q_{I A}-C_{I K} Q_{J A} \\
{\left[L_{A B}, Q_{I C}\right] } & =-\mathcal{J}_{B C} Q_{I A}-\mathcal{J}_{A C} Q_{I B} \\
\left\{Q_{I A}, Q_{J B}\right\} & =C_{I J} L_{A B}-\mathcal{J}_{A B} R_{I J}
\end{aligned}
$$

where $I, J=1, \cdots, 8$ are spinor indices for chiral subspace of so(2,6) and thus $(1+5)$-dimensional spinor indices [36]. The charge conjugation matrix $C_{I J}$ is symmetric, $C_{I J}=C_{J I}$. The indices $A, B=1, \cdots, 4$ are for usp(4) and $\mathcal{J}_{A B}$ is the symplectic form. The anti-symmetric matrix $R_{I J}$ generates so(2,6). The symmetric matrix $L_{A B}$ generates usp(4).

Let us decompose the index $I$ into $(a, \dot{a})$ with $a=1, \cdots, 4$ and $\dot{a}=\dot{1}, \cdots, \dot{4}$. Then the related quantities are rewritten as [37].

$$
\begin{aligned}
& R_{I J}=\left(\begin{array}{cc}
P_{a b} & J_{a \dot{b}}+\frac{1}{2} c_{a \dot{b}} D \\
J_{\dot{a} b}-\frac{1}{2} c_{\dot{a} b} D & K_{\dot{a} \dot{b}}
\end{array}\right), \quad C_{I J}=\left(\begin{array}{cc}
0 & c \\
c^{T} & 0
\end{array}\right) \\
& D=\frac{1}{2} c_{a \dot{b}} R_{a \dot{b}}, \quad Q_{I A}=\left(Q_{a A}, S_{\dot{a} A}\right), \quad c_{a \dot{b}} J_{a \dot{b}}=0
\end{aligned}
$$

We use here the spinor basis in which

$$
c_{a \dot{b}}=\left(\begin{array}{cc}
0 & -\epsilon \\
-\epsilon & 0
\end{array}\right), \quad \epsilon=\left(\begin{array}{cc}
0 & 1 \\
-1 & 0
\end{array}\right)
$$


Note that $c_{a \dot{b}} c_{\dot{b} c}=\delta_{a c}$ and $c_{\dot{b} a}=c_{a \dot{b}}$.

It is straightforward to rewrite the (anti-)commutation relations of $\operatorname{osp}\left(8^{*} \mid 4\right)$ in terms of these generators.

- $\operatorname{so}(1,5) \quad J_{a \dot{b}} \quad\left(-J_{1 \dot{4}}+J_{2 \dot{3}}-J_{3 \dot{2}}+J_{4 \dot{1}}=0\right) \quad 15$

$$
\left[J_{a \dot{b}}, T_{c}\right]=c_{\dot{b} c} T_{a}-\frac{1}{4} c_{a \dot{b}} T_{c}, \quad\left[J_{a \dot{b}}, T_{\dot{c}}\right]=-c_{a \dot{c}} T_{\dot{b}}+\frac{1}{4} c_{a \dot{b}} T_{\dot{c}}
$$

- $\mathrm{usp}(4) \simeq \operatorname{so}(5) \quad L_{A B} \quad 10$

$$
\left[L_{A B}, T_{C}\right]=-\mathcal{J}_{B C} T_{A}-\mathcal{J}_{A C} T_{B}
$$

- dilatation $D \quad 1$

$$
[D, T]=d(T) T \quad \begin{array}{|c|c|c|c|c|c|}
\hline & K_{\dot{a} \dot{b}} & S_{\dot{a} A} & D, J_{a \dot{b}}, L_{A B} & Q_{a A} & P_{a b} \\
\hline d & -1 & -1 / 2 & 0 & 1 / 2 & 1 \\
\hline
\end{array}
$$

- supertranslation $Q_{a A}$ and superconformal symmetry $S_{\dot{a} A} \quad 16+16$

$$
\begin{aligned}
& \left\{Q_{a A}, Q_{b B}\right\}=-\mathcal{J}_{A B} P_{a b}, \quad\left\{S_{\dot{a} A}, S_{\dot{b} B}\right\}=-\mathcal{J}_{A B} K_{\dot{a} \dot{b}} \\
& \left\{Q_{a A}, S_{\dot{b} B}\right\}=c_{a \dot{b}} L_{A B}-\mathcal{J}_{A B}\left(J_{a \dot{b}}+\frac{1}{2} c_{a \dot{b}} D\right)
\end{aligned}
$$

- translation $P_{a b}\left(=-P_{b a}\right)$ and special conformal transformation $K_{\dot{a} \dot{b}}\left(=-K_{\dot{b} \dot{a}}\right) \quad 6+6$

$$
\begin{aligned}
& {\left[P_{a b}, S_{\dot{c} A}\right]=c_{b \dot{c}} Q_{a A}-c_{a \dot{c}} Q_{b A}, \quad\left[K_{\dot{a} \dot{b}}, Q_{c A}\right]=c_{\dot{b} c} S_{\dot{a} A}-c_{\dot{a} c} S_{\dot{b} A}} \\
& {\left[P_{a b}, K_{\dot{c} \dot{d}}\right]=c_{b \dot{c}} J_{a \dot{d}}-c_{a \dot{c}} J_{b \dot{d}}-c_{b \dot{d}} J_{a \dot{c}}+c_{a \dot{d}} J_{b \dot{c}}+\left(c_{b \dot{c}} c_{a \dot{d}}-c_{a \dot{c}} c_{b \dot{d}}\right) D}
\end{aligned}
$$

\section{References and Notes}

1. Maldacena, J.M. The large N limit of superconformal field theories and supergravity. Adv. Theor. Math. Phys. 1998, 2, 231-252.

2. Gubser, S.S.; Klebanov, I.R.; Polyakov, A.M. Gauge theory correlators from non-critical string theory. Phys. Lett. B 1998, 428, 105-114.

3. Witten, E. Anti-de Sitter space and holography. Adv. Theor. Math. Phys. 1998, 2, 253-291.

4. Hartnoll, S.A. Lectures on holographic methods for condensed matter physics, Class. Quant. Grav. 2009, 26, 224002:1-224002:61.

5. Sachdev, S. Condensed matter and AdS/CFT. Lect. Notes Phys. 2011, 828, 273-311.

6. Son, D.T. Toward an AdS/cold atoms correspondence: A Geometric realization of the Schrodinger symmetry. Phys. Rev. D 2008, 78, 046003:1-046003:7.

7. Balasubramanian, K.; McGreevy, J. Gravity duals for non-relativistic CFTs. Phys. Rev. Lett. 2008, 101, 061601:1-061601:4. 
8. Kachru, S.; Liu, X.; Mulligan, M. Gravity Duals of Lifshitz-like Fixed Points, Phys. Rev. D 2008, 78, 106005:1-106005:8.

9. Schafer-Nameki, S.; Yamazaki, M.; Yoshida, K. Coset Construction for Duals of Non-relativistic CFTs. J. High Energy Phys. 2009, doi: 10.1088/1126-6708/2009/05/038.

10. Hagen, C.R. Scale and conformal transformations in galilean-covariant field theory, Phys. Rev. D 1972, 5, 377-388.

11. Niederer, U. The maximal kinematical invariance group of the free Schrodinger equation. Helv. Phys. Acta 1972, 45, 802-810.

12. Henkel, M. Schrodinger invariance in strongly anisotropic critical systems. J. Statist. Phys. 1994, 75, 1023-1061.

13. Nishida, Y.; Son, D.T. Nonrelativistic conformal field theories. Phys. Rev. D 2007, 76, 086004:1-086004:14.

14. Duval, C.; Horvathy, P.A. On Schrodinger superalgebras. J. Math. Phys. 1994, 35, 2516-2538.

15. Henkel, M.; Unterberger, J. Supersymmetric extensions of Schrödinger invariance. Nucl. Phys. B 2006, 746, 155-201.

16. Sakaguchi, M.; Yoshida, K. Super Schrödinger algebra in AdS/CFT. J. Math. Phys. 2008, 49, 102302:1-102302:13.

17. Sakaguchi, M.; Yoshida, K. More super Schrodinger algebras from psu(2,2|4). J. High Energy Phys. 2008, doi: 10.1088/1126-6708/2008/08/049.

18. Maldacena, J.M.; Martelli, D.; Tachikawa, Y. Comments on string theory backgrounds with non-relativistic conformal symmetry. J. High Energy Phys. 2008, doi: 10.1088/1126-6708/ 2008/10/072.

19. Hartnoll, S.A.; Yoshida, K. Families of IIB duals for nonrelativistic CFTs. J. High Energy Phys. 2008, doi: 10.1088/1126-6708/2008/12/071.

20. Donos, A.; Gauntlett, J.P. Supersymmetric solutions for non-relativistic holography. J. High Energy Phys. 2009, doi: 10.1088/1126-6708/2009/03/138.

21. Donos, A.; Gauntlett, J.P. Solutions of type IIB and $D=11$ supergravity with Schrodinger(z) symmetry. J. High Energy Phys. 2009, doi: 10.1088/1126-6708/2009/07/042.

22. Ooguri, H.; Park, C.-S. Supersymmetric non-relativistic geometries in M-theory. Nucl. Phys. B 2010, 824, 136-153.

23. Donos, A.; Gauntlett, J.P. Schrodinger invariant solutions of type IIB with enhanced supersymmetry. J. High Energy Phys. 2009, doi: 10.1088/1126-6708/2009/10/073.

24. Bobev, N.; Kundu, A. Deformations of Holographic Duals to Non-Relativistic CFTs. J. High Energy Phys. 2009, doi: 10.1088/1126-6708/2009/07/098.

25. Bobev, N.; Kundu, A.; Pilch, K. Supersymmetric IIB Solutions with Schrodinger Symmetry. $J$. High Energy Phys. 2009, doi: 10.1088/1126-6708/2009/07/098.

26. Liu, H.-S.; Lu, H. Supersymmetry of the Schrodinger and PP wave solutions in Einstein-Weyl supergravities. 2012, arXiv:1206.4371.

27. Balasubramanian, K.; Narayan, K. Lifshitz spacetimes from AdS null and cosmological solutions. J. High Energy Phys. 2010, arXiv:1005.3291v3. 
28. Donos, A.; Gauntlett, J.P. Lifshitz Solutions of D = 10 and D = 11 supergravity. J. High Energy Phys. 2010, doi: 10.1007/JHEP12(2010)002.

29. Gregory, R.; Parameswaran, S.L.; Tasinato, G.; Zavala, I. Lifshitz solutions in supergravity and string theory. J. High Energy Phys. 2010, doi: 10.1007/JHEP12(2010)047.

30. Cassani, D.; Faedo, A.F. Constructing Lifshitz solutions from AdS. J. High Energy Phys. 2011, doi: 10.1007/JHEP05(2011)013.

31. Halmagyi, N.; Petrini, M.; Zaffaroni, A. Non-Relativistic Solutions of N=2 Gauged Supergravity. J. High Energy Phys. 2011, doi: 10.1007/JHEP08(2011)041.

32. Narayan, K. Lifshitz-like systems and AdS null deformations. Phys. Rev. D 2011, 84, 086001:1-086001:10.

33. Chemissany, W.; Hartong, J. From D3-Branes to Lifshitz Space-Times. Class. Quant. Grav. 2011, 28, 195011:1-195011:13.

34. Beisert, $\mathrm{N}$. The dilatation operator of $\mathrm{N}=4$ super Yang-Mills theory and integrability. Phys. Rept. 2005, 405, 1-202.

35. The triality of $\mathrm{SO}(8)$ and hence of $\mathrm{SO}^{*}(8)$ makes this possible. Concretely, we take $\Omega=\left(\begin{array}{cc}C_{I J} & 0 \\ 0 & i \mathcal{J}_{A B}\end{array}\right)$.

36. Claus, P.; Kallosh, R.; Van Proeyen, A. M 5-brane and superconformal $(0,2)$ tensor multiplet in 6 dimensions, Nucl. Phys. B 1998, 518, 117-150.

37. A chiral spinor of $\mathrm{SO}(2,6)$ is decomposed into $4+\overline{4}$ under $\mathrm{SU}_{c}^{*}(4)$.

(c) 2012 by the authors; licensee MDPI, Basel, Switzerland. This article is an open access article distributed under the terms and conditions of the Creative Commons Attribution license (http://creativecommons.org/licenses/by/3.0/). 